\title{
Sulodexide may alleviate neointimal hyperplasia by inhibiting angiopoietin-2 in an arteriovenous fistula model
}

\author{
YAN LEI, ZHIHUA ZHENG, YING WANG, YUYUN LIU, RONGJUN LIU, QINGDONG XU and XUEQING YU

\begin{abstract}
Department of Nephrology, The First Affiliated Hospital, Sun Yat-Sen University and Key Laboratory of Nephrology,
\end{abstract} \\ Ministry of Health, Guangzhou 510080, P.R. China
}

Received September 20, 2012; Accepted December 5, 2012

DOI: $10.3892 / \mathrm{mmr} .2013 .1293$

\begin{abstract}
The present study was undertaken to confirm whether sulodexide aleviates neointimal hyperplasia by regulating angiopoietin/Tie in a rat femoral arteriovenous fistula (AVF) model. Sprague Dawley rats were divided into four groups: sham, model, treatment and treatment control. An arteriovenous shunt model was created in the model and treatment groups. Sulodexide was subcutaneously administered (10 mg/kg/day) 6 times per week for 8 weeks in the treatment and treatment control groups. Histology and immunofluorescence were analyzed and the protein expression of angiopoietin-1, angiopoietin-2, Tie-2, p-ERK and total-ERK were tested by ELISA and/or western blotting after 8 weeks. HE staining revealed that sulodexide was able to partially alleviate intimal hyperplasia of remodeled veins in the AVF model. Additionally, sulodexide was able to decrease angiopoietin-2 and Tie-2 expression while increasing angiopoietin-1 expression in AVF tissue. Sulodexide was also able to decrease ERK phosphorylation which was increased in the model. Serum levels of soluble Tie-2 (sTie-2) were also significantly decreased by sulodexide compared with the model. Immunofluorescent analysis also confirmed that sulodexide was able to decrease angiopoietin-2 expression, possibly partially by inhibiting endothelial cell proliferation. Sulodexide may alleviate venous intimal hyperplasia by regulating the angiopoietin/Tie system, which may play a significant role in assisting remodeled veins to cope with their new biomechanical environment, but whether the angiopoietin/Tie system is beneficial or not requires further study.
\end{abstract}

\section{Introduction}

In hemodialysis (HD) patients, vascular access (VA) is a requirement for life and according to the K/DOQI guidelines,

Correspondence to: Professor Zhihua Zheng, Department of Nephrology, The First Affiliated Hospital of Sun Yat-sen University, 58 Zhongshan Road II, Guangzhou 510080, P.R. China

E-mail: zhihuazheng@126.com

Key words: sulodexide, arteriovenous fistula, angiogenesis, angiopoietin-1, angiopoietin-2, Tie-2 an arteriovenous fistula (AVF) is the first choice of VA for nephrologists. However, HD VA dysfunction affects the long-term survival of dialysis patients as it is a major cause of inadequate dialysis and cardiovascular disease (CVD) morbidity $(1,2)$. The mechanism behind VA failure is considered to be neointimal hyperplasia of the vascular smooth muscle cells near the venous anastomosis of the AVFs, resulting in vein wall thickening, stenosis and, ultimately, occlusion (3). Chronic kidney disease (CKD) in itself also accelerates the development of neointimal hyperplasia at the anastomotic site of an AVF (4,5).

In previous years, a number of studies revealed that the pathogenesis and manifestation of AVFs are similar to those of atherosclerosis and that angiogensis occurs during typical neointimal hyperplasia of the AVF (6-8). Angiogenesis was thought to depend on a precise balance of positive and negative regulation (9). Angiopoietin-1 and angiopoietin-2 are antagonistic non-redundant gatekeepers of endothelial activation and thus are potentially significant factors in accelerated atherosclerosis (9-11). Angiopoietin/Tie signaling is essential during embryonic vessel assembly and maturation, as well as in functioning as a key regulator of adult vascular homeostasis (12). In recent years, the angiopoietin/Tie system, and angiopoietin-2 in particular, has emerged as a predictive marker for cardiovascular risk in hypertension, rheumatoid arthritis, dialysis and congestive heart failure patients (10,13-15).

Sulodexide (KRX-101) is a mixture of GAGs composed of $80 \%$ low-molecular mass heparin and $20 \%$ dermatan sulfate (16). Sulodexide is effective in partially reversing the thrombogenic coagulation profile without increasing the risk of bleeding (17). Sulodexide has primarily been used in treating peripheral occlusive arterial disease (18) and more recently to reduce albumin excretion rates and improve microvasular function of diabetic nephropathy. Sulodexide has also had a marked effect on plasma viscosity and plasma fibrinogen concentrations (19). A previous study revealed that it may be an effective adjunctive agent in myocardial revascularization procedures as it is able to reduce myocardial infarct size and the serum concentration of troponin I during reperfusion (16).

Angiogenesis is implicated in endothelial and cardiac injury. The present study provides a novel description and characterization of neoangiogenesis occurring in the venous limb of an AVF in the rat model which, as we previously demonstrated, exhibits intimal hyperplasia and proinflammatory 
changes (20-23). In the present study, the possible role of sulodexide in treating the AVF model was tested to observe if there are any changes in the fistula tissue. Angiopoietin-1, angiopoietin-2 and Tie-2 expression were also emphasized in the angiogenesis process implicated in the rat femoral AVF model.

\section{Subjects and methods}

Subjects. All experiments were approved by the local animal humane board and were performed in accordance with Chinese legislation on the protection of animals. Male 12-week-old Sprague Dawley rats (250-300 g) were purchased from a commercial breeder (Guangdong Medical Laboratory Animal Center, Guangdong, China). The rats were kept in a climate-controlled room $\left(21^{\circ} \mathrm{C}\right.$ and $60 \%$ relative humidity) with a 12-h cycle of light and darkness. All animals were housed in normal cages with free access to water and food.

Surgery. Rats were anesthetized by sodium phenobarbital $(60 \mathrm{mg} / \mathrm{kg})$ and placed into a supine position on a heating pad (TR-200, Fine Science Tools, Heidelberg, Germany) prior to performing an end-to-side anastomosis of the femoral artery to the femoral vein. The femoral vasculature was exposed by a $2-\mathrm{cm}$ incision along the left inguinal fold, and by retraction of the soft tissues and abdominal musculature. The femoral artery and vein were freed from the surrounding fascia and the femoral nerve by careful dissection with the aid of a dissecting scope (Nikon Instruments, Melville, NY, USA; x10 to x16 magnification). The branching vessels from the femoral artery and vein were ligated doubly with sterile 6-0 silk sutures and then divided. The artery was ligated at the distal end of its exposure, clamped with a non-traumatic aneurysm clip at the proximal end and transected just proximal to the ligation at a $45^{\circ}$ angle. Approximating clamps were situated on the vein, framing the site of the anastomosis and a small longitudinal incision was made with a microsurgical knife. The lumina of the two vessels were rinsed with heparinized saline and the transected end of the artery was attached to the opening in the adjacent vein using 11-0 monofilament Ethilon nylon sutures (Ethicon, Shanghai, China) to make eight equidistant interrupted sutures. The approximating clamps and the aneurysm clip were then removed and the arterial flow into the femoral vein was verified by a visual inspection. Finally, the femoral vein was ligated just distal to the anastomosis and the skin was closed with 3-0 continuous sutures. In a similar fashion, sham surgeries, consisting of the inguinal incision and dissection of the vasculature from the surrounding tissue, were performed on the control rats. At 8 weeks post-surgery, the rats were euthanized for the harvest of the vasculature of the AVF and to collect the femoral arteries and veins from rats that underwent sham surgeries.

Study design. The rats were divided into 4 groups, the sham $(n=6)$, model $(n=6)$, treatment $(n=6)$ and treatment control groups $(\mathrm{n}=6)$. Sulodexide $(10 \mathrm{mg} / \mathrm{kg})$ was injected subcutaneously six times a week in the treatment and treatment control groups. Rats underwent the AVF surgery in the model and treatment groups and were sacrificed 8 weeks later.
Measurements of systemic concentrations of cytokines. Serum levels of angiopoietin-1, angiopoietin-2 and soluble Tie-2 (sTie-2) were determined by ELISA kits (catalog no. DANG10, DANG20 and DTE200, respectively).

\section{Histology and immunoflurescence}

Cardiac perfusion and tissue preparation. Prior to tissue harvesting, anesthesia was administered as described previously. The groinal incisions were followed by cardiac puncture to flush the vessels, first with a saline solution and then with $10 \%$ neutral formalin in phosphate-buffered saline (PBS, pH 7.4). The femoral veins from the four groups were gently removed and fixed in 10\% neutral formalin in PBS for histological and immunofluorescent analysis. Following overnight fixation, the specimens were processed and embedded in paraffin using standard techniques. The $4-\mu \mathrm{m}$ longitudinal sections were stained with hematoxylin-eosin (HE) for morphometrical analysis and the 6- $\mu \mathrm{m}$ sections stained with $\mathrm{HE}$ for immunofluorescent analysis at the site of the anastomosis. The rest of tissues were stored at $-80^{\circ} \mathrm{C}$ for further study.

Western blot analysis. Western blot analysis was performed as described in the literature (8). Briefly, proteins (25-60 $\mu \mathrm{g})$ were separated on $10 \%$ Tris- $\mathrm{HCl}$ gels (Bio-Rad, Hercules, CA, USA) and transferred to nitrocellulose membranes. Primary antibodies for angiopoietin-1, angiopoietin-2, Tie-2, vWF (catalog no. 612392, 610296 and 610431, respectively; Abcam, Cambridge, MA, USA) or $\beta$-actin (catalog no. 2118; Cell Signaling Technology Inc., Danvers, MA, USA) were used in overnight incubations at $4^{\circ} \mathrm{C}$. Horseradish peroxidase-conjugated secondary antibodies were then used and bands were visualized using an enhanced chemiluminescence method.

Immunofuorescence analysis. Immunofluorescence was performed using commercial antibodies. Standard immunofuorescence protocols were used; deparafinization and hydration was followed by antigen demasking (2\% citrate buffer in an autoclave) and nonspecific protein binding with $10 \%$ fetal goat serum in 3\% BSA (TBS) buffer for $60 \mathrm{~min}$. Primary antibody incubation took place overnight at $4{ }^{\circ} \mathrm{C}$ and at room temperature the next day. Secondary antibody blend incubation followed for $60 \mathrm{~min}$ at room temperature and subsequent to washing with PBS 3 times, another primary antibody was incubated for $60 \mathrm{~min}$ followed by the relevent secondary antibody. Subsequent to this, DAPI was used to stain the nuclei for $7 \mathrm{~min}$ and then samples were embedded using a mounting medium.

Statistical analysis. Data are expressed as mean \pm SEM. The Student's t-test and ANOVA were used for comparisons between the groups. $\mathrm{P}<0.05$ was considered to indicate a statistically significant difference.

\section{Results}

General characteristics. AVF model rats were made by using an end-to-side anastomosis of the femoral artery to the femoral vein. There were no observable procedure-related complications. All animals survived the procedures and were sacrificed at 8 weeks post-surgery. During the observation period in between, there was no sign of any peripheral ischemia resulting from steal syndrome or edema caused by venous congestion. 

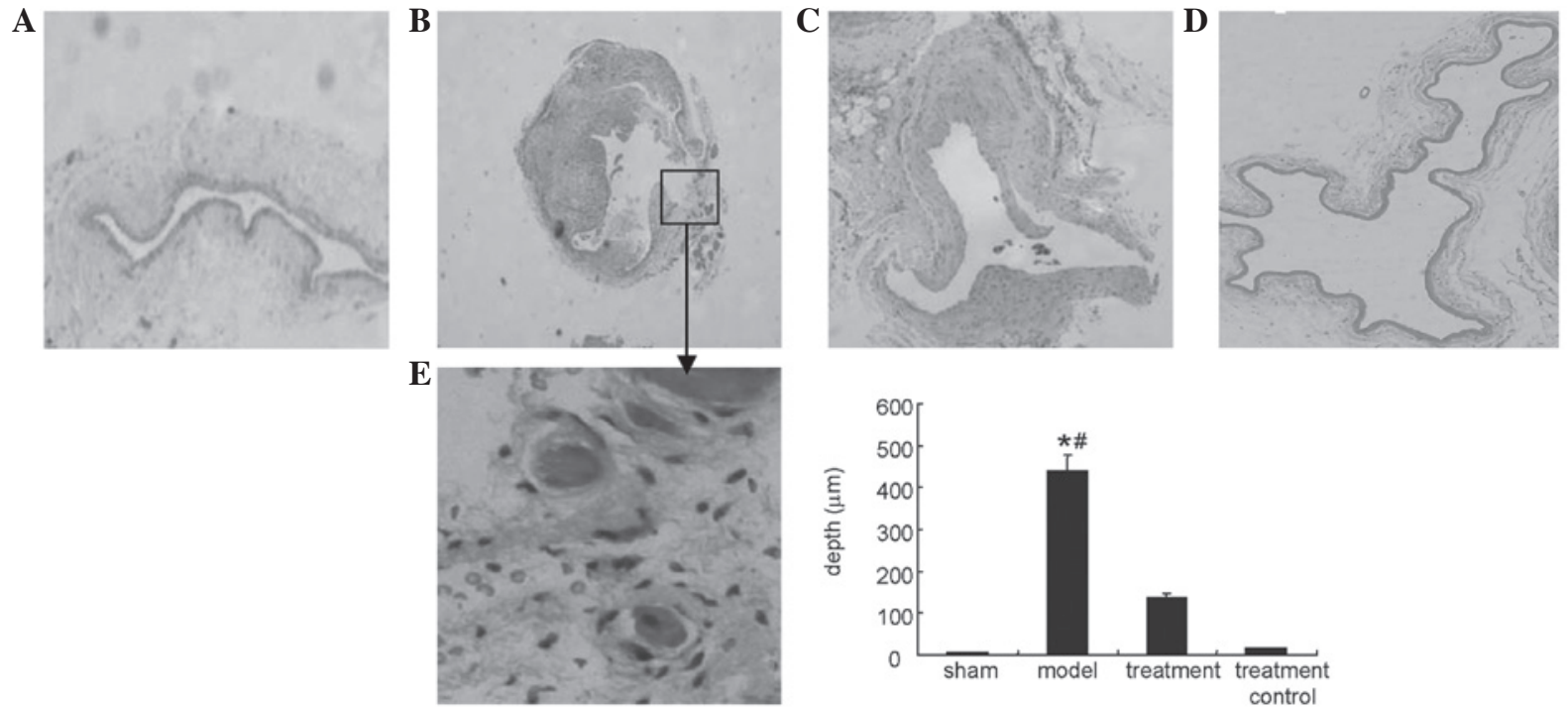

Figure 1. Histology of the venous limb of an arteriovenous fistula (AVF) and femoral vein. Low-power light microscopy (x100 magnification). (A) Femoral vein from sham surgery. (B) Remodeled vein. (C) Remodeled vein following sulodexide treatment (D) Sulodexide treatment control. (E) High-power light microscopy (x400 magnification) revealing angiogenesis at the base of the venous limb of the AVF. Sections are stained with hematoxylin and eosin. The depth of the remodeled vein was decreased by sulodexide treatment $(\mathrm{P}<0.05)$. ${ }^{*} \mathrm{P}<0.05$ vs. sham group; ${ }^{~} \mathrm{P}<0.05$ vs. treatment group.
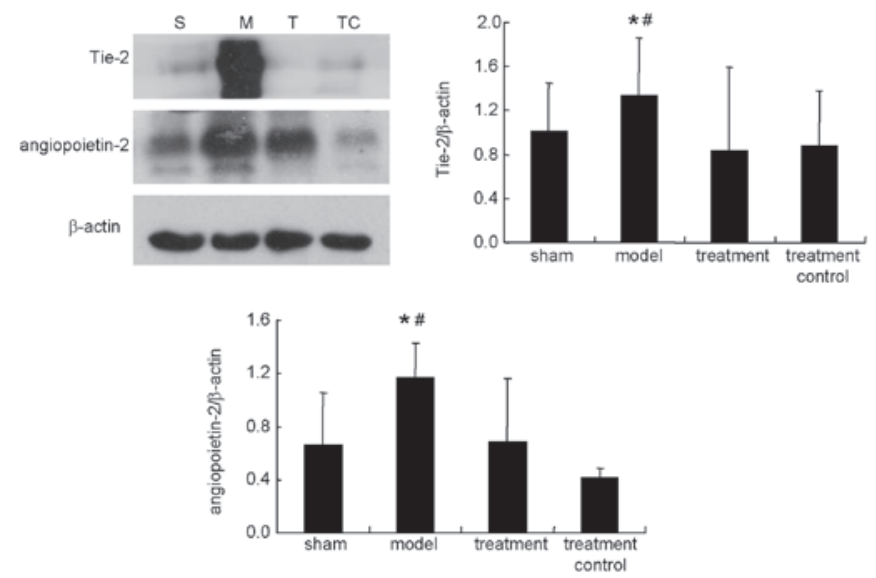

Figure 2. Expression of Tie-2 and angiopoietin-2 protein in the venous limb of the rat arteriovenous fistula (AVF) and in femoral veins from sham rats at 8 weeks after AVF or sham surgery. Equivalency of loading was assessed by immunoblotting for $\beta$-actin. ${ }^{*} \mathrm{P}<0.05$ vs. sham group; ${ }^{*} \mathrm{P}<0.05$ vs. treatment group. S, sham group; M, model group; T, treatment group; TC, treatment control group.

Descriptive histology. Fig. 1 shows intimal hyperplasia of the venous limb as identified in the model and treatment groups. Histological analysis revealed that intimal hyperplasia was irregular with marked cellular proliferation and with associated angiogenesis in certain areas. The depth of the intima was decreased in the treatment group in comparison to that of model group.

ELISA analysis. Serum levels of angiopoietin-1 and angiopoietin-2 were not detected by ELISA in the present study. However, serum sTie-2 levels which were increased in the AVF model group were significantly decreased in the sulodexide treatment group $(\mathrm{P}<0.05)$. There was also a significant difference in serum sTie-2 levels between the treatment and treatment control groups $(\mathrm{P}<0.05)$, as depicted in Table I.
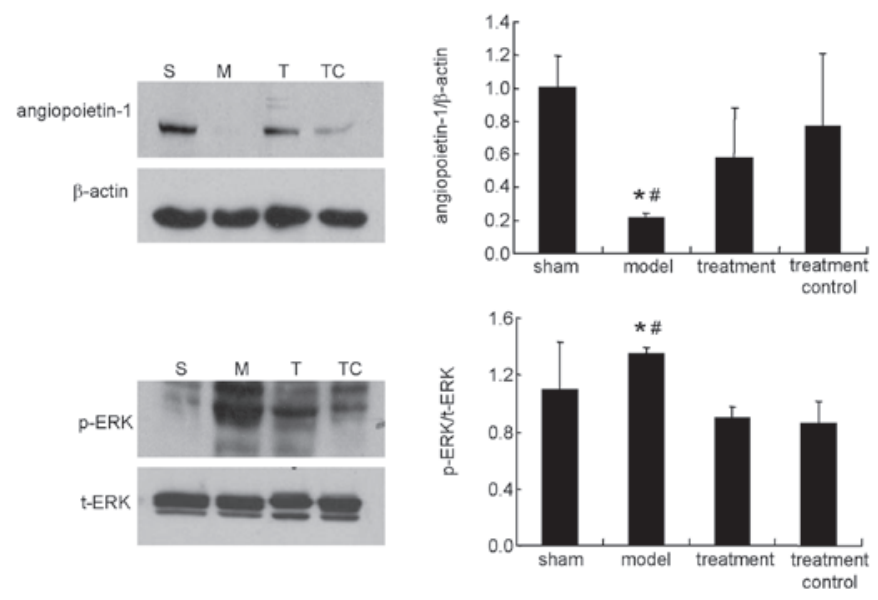

Figure 3. Expression of angiopoietin-1 and p-ERK/t-ERK protein in the venous limb of the rat arteriovenous fistula (AVF) and in femoral veins from sham rats at 8 weeks after AVF or sham operation. Equivalency of loading was assessed by immunoblotting for $\beta$-actin. ${ }^{*} \mathrm{P}<0.05$ vs. sham group; ${ }^{\#} \mathrm{P}<0.05$ vs. treatment group. S, sham group; M, model group; T, treatment group; TC, treatment control group.

Western blot. As shown in Figs. 2 and 3, sulodexide decreased the expression of angiopoietin-2 and Tie-2, which were upregulated in the model $(\mathrm{P}<0.05)$, and increased the expression of angiopoietin-1, which was downregulated in the model $(\mathrm{P}<0.05)$. Sulodexide also inhibited the phosphorylation of ERK, which was increased in the fistula tissue, when compared with tissue from the sham group $(\mathrm{P}<0.05)$. There was no significant difference between the sham and treatment control groups $(\mathrm{P}>0.05)$.

Immunoflorescent analysis. As shown in Fig. 4, sulodexide was able to decrease angiopoietin-2 expression which was markedly elevated in the model group. Angiopoietin-2 was colocalized with vWF, an endothelial cell marker. It is possible that sulodexide decreased angiopoietin-2 expression by inhibiting endothelial cell proliferation to a certain extent. 
Table I. Serum concentrations of sTie-2 in the four groups (ng/ml).

\begin{tabular}{lccccr}
\hline Variable & $\begin{array}{c}\text { Sham group } \\
(\mathrm{n}=6)\end{array}$ & $\begin{array}{c}\text { Model group } \\
(\mathrm{n}=6)\end{array}$ & $\begin{array}{c}\text { Treatment group } \\
(\mathrm{n}=6)\end{array}$ & $\begin{array}{c}\text { Treatment control group } \\
(\mathrm{n}=6)\end{array}$ & P-value \\
\hline sTie-2 & $0.030 \pm 0.010$ & $0.094 \pm 0.034$ & $0.055 \pm 0.022$ & $0.029 \pm 0.005$ & 0.015 \\
\hline
\end{tabular}
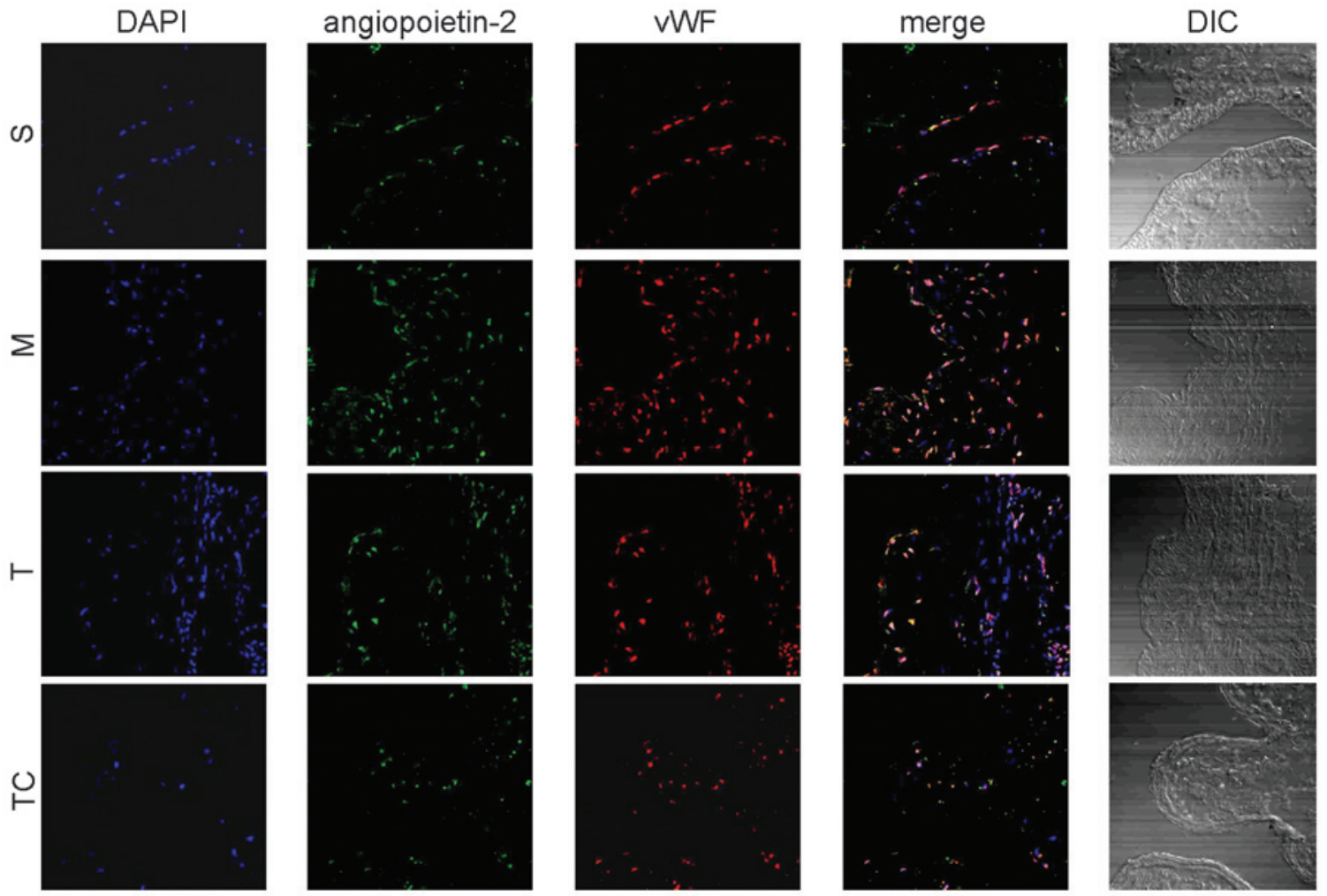

Figure 4. Immunoflorescent analysis of angiopoietin-2 and vWF expression in the venous limb of the rat arteriovenous fistula (AVF) and in the femoral veins from sham rats at 8 weeks subsequent to AVF or sham surgeries. Angiopoietin-2 and vWF were colocalized in the intima of the vein (x630 magnification)..S, sham group; M, model group; T, treatment group; TC, treatment control group; DIC, differential interference contrast.

\section{Discussion}

In an AVF, the venous vasulcature is exposed to the arterial circulation and the veins are subjected to hemodynamic stresses that they were not designed to cope with. This results in venous intimal hyperplasia (7).

Oxygen and nutrient diffusion out of the veins and arteries is impeded by hypertrophy and hyperplasticity. Angiogenesis is able to circumvent this, but it may also promote atherosclerotic plaque progression and ruptures. Neoangiogenesis thus generates pathogenetically significant lesions and is the focus of numerous investigations which aim to elucidate the role of angiogenesis in hemodynamic change-induced cardiovascular remodeling following the creation of an AVF.

Sulodexide is used for the prophylaxis and treatment of thromboembolic diseases (peripheral vascular diseases, PVDs), however, a recent study has also demonstrated the beneficial effects of sulodexide in animal models of reperfusion injury (16) and the treatment of diabetic nephropathy (20), suggesting it has cardiovascular protection properties. In the present study, sulodexide was used to treat an animal model of an AVF. Intimal hyperplasia was alleviated significantly by sulodexide and a possible role for the drug was implicated in the process.

Previous studies have demonstrated that angiopoietin-2/ Tie-2 is part of the molecular response to shear stress which may regulate angiogenesis. Therefore, the present study measured the concentration of angiogenic species, angiopoietin-1, angiopoietin-2 and sTie-2 in the circulation. However, angiopoietin-1 and angiopoietin-2 levels were undetectable in the serum, while sTie-2 upregulation was observed in the AVF model group and downregulation occurred following sulodexide treatment.

The present study also examined whether the expression of angiopoietin-1, angiopoietin-2 and Tie-2 was induced within the venous limbs of the AVF. The results showed that protein expression of angiopoietin-2 and Tie-2 was upregulated while angiopoietin-1 was downregulated in the model. Following sulodexide treatment, angiopoietin-2 expression was downregulated and angiogenesis was decreased in the AVF. Angiopoietin-2 participates in flow-dependent vascular adaptation (22) and its expression may be increased by activation of the AMP-activated protein kinase induced by shear stress (23). The upregulation of angiopoietin-2 may be a result 
of angiogenesis responding to the unusual increased blood flow. In models characterized by vascular injury, another study has also demonstrated that sulodexide is able to inhibit intima proliferation in the carotid artery (24).

The present study extends these observations by demonstrating that upregulation of vWF also occurs in the venous circulation when it is subjected to an increased blood flow. Angiopoietin-2 and vWF antibody were also used for immunofluorescent analysis to determine the phenotype of these angiopoietin-2-positive cells using laser confocal microscopy. A colabeling technique was employed that also probed for the presence of a marker of the endothelial cells (vWF). Angiopoietin-2 and vWF were identified as colocalized in the cytoplasm of the endothelial cells. This showed that sulodexide was able to decrease angiopoietin-2 expression which was increased in the AVF. Endothelial cell proliferation was clearly observed in the venous limbs of the AVF. It is possible that sulodexide decreases the angiopoietin-2 expression by inhibiting endothelial cell proliferation to a certain extent.

In the present study, sulodexide decreased intimal hyperplasia, possibly through regulation of the angiopoietin/ Tie system, which is dysregulated in the process of intimal hyperplasia induced by venous hypertension. Further study of the details of this mechanism are required. To a certain extent, this study may provide therapeutic prospects for intimal hyperplasia of AVF in the future.

\section{Acknowledgements}

The authors would like to thank Dr Liu from the Department of Vascular Surgery for his assistance in making the AVF model and also Professor Huang Xi from the Department of Immunology, Sun Yat-sen University, for his assistance with the experiment. This study was financially supported by the Natural Science Foundation of Guangdong Province, P.R. China (2012B031800448).

\section{References}

1. Manca O, Pisano GL, Carta P, et al: The management of hemodialysis arteriovenous fistulas in well functioning renal transplanted patients: many doubts, few certainties. J Vasc Access 6: 182-186, 2005.

2. Lee T and Roy-Chaudhury P: Advances and new frontiers in the pathophysiology of venous neointimal hyperplasia and dialysis access stenosis. Adv Chronic Kidney Dis 16: 329-338, 2009.

3. Langer S, Heiss C, Paulus N, et al: Functional and structural response of arterialized femoral veins in a rodent AV fistula model. Nephrol Dial Transplant 24: 2201-2206, 2009.

4. Kokubo T, Ishikawa N, Uchida H, et al: CKD accelerates development of neointimal hyperplasia in arteriovenous fistulas. J Am Soc Nephrol 20: 1236-1245, 2009.

5. Langer S, Kokozidou M, Heiss C, et al: Chronic kidney disease aggravates arteriovenous fistula damage in rats. Kidney Int 78: $1312-1321,2010$.
6. Rodríguez-Pla A, Bosch-Gil JA, Rosselló-Urgell J, Huguet-Redecilla P, Stone JH and Vilardell-Tarres M: Metalloproteinase-2 and -9 in giant cell arteritis: involvement in vascular remodeling. Circulation 112: 264-269, 2005.

7. Caplice NM, Wang S, Tracz M, et al: Neoangiogenesis and the presence of progenitor cells in the venous limb of an arteriovenous fistula in the rat. Am J Physiol Renal Physiol 293: F470-F475, 2007.

8. Kanwar YS: Functional duality of progenitor cells influxing into arteriovenous fistula during its neoangiogenesis. Am J Physiol Renal Physiol 293: F468-F469, 2007.

9. Maisonpierre PC, Suri C, Jones PF, et al: Angiopoietin-2, a natural antagonist for Tie2 that disrupts in vivo angiogenesis. Science 277: 55-60, 1997.

10. David S, Kümpers P, Hellpap J, et al: Angiopoietin 2 and cardiovascular disease in dialysis and kidney transplantation. Am J Kidney Dis 53: 770-778, 2009.

11. Vandenbunder B: Angiopoietin-2, a new molecular actor involved in vascular tree morphogenesis. Bull Cancer 84: 1079-1080, 1997 (In French).

12. Augustin HG, Koh GY, Thurston G and Alitalo K: Control of vascular morphogenesis and homeostasis through the angiopoietin-Tie system. Nat Rev Mol Cell Biol 10: 165-177, 2009.

13. Chong AY, Caine GJ, Freestone B, Blann AD and Lip GY: Plasma angiopoietin-1, angiopoietin-2, and angiopoietin receptor tie-2 levels in congestive heart failure. J Am Coll Cardiol 43: 423-428, 2004.

14. Patel JV, Lim HS, Varughese GI, Hughes EA and Lip GY: Angiopoietin-2 levels as a biomarker of cardiovascular risk in patients with hypertension. Ann Med 40: 215-222, 2008.

15. Westra J, de Groot L, Plaxton SL, et al: Angiopoietin-2 is highly correlated with inflammation and disease activity in recent-onset rheumatoid arthritis and could be predictive for cardiovascular disease. Rheumatology (Oxford) 50: 665-673, 2011.

16. Lauver DA, Booth EA, White AJ, Poradosu E and Lucchesi BR: Sulodexide attenuates myocardial ischemia/reperfusion injury and the deposition of C-reactive protein in areas of infarction without affecting hemostasis. J Pharmacol Exp Ther 312: 794-800, 2005.

17. Kim SB, Kim SH, Lee MS, Chang JW, Lee SK and Park JS: Effects of sulodexide on hemostatic factors, lipid profile, and inflammation in chronic peritoneal dialysis patients. Perit Dial Int 27: 456-460, 2007.

18. Shustov SB: Controlled clinical trial on the efficacy and safety of oral sulodexide in patients with peripheral occlusive arterial disease. Curr Med Res Opin 13: 573-582, 1997.

19. Lunetta $M$ and Salanitri T: Lowering of plasma viscosity by the oral administration of the glycosaminoglycan sulodexide in patients with peripheral vascular disease. J Int Med Res 20: 45-53, 1992.

20. Rossini M, Naito T and Yang H, et al: Sulodexide ameliorates early but not late kidney disease in models of radiation nephropathy and diabetic nephropathy. Nephrol Dial Transplant 25: 1803-1810, 2010.

21. Lin T, Horsfield C and Robson MG: Arteriovenous fistula in the rat tail: a new model of hemodialysis access dysfunction. Kidney Int 74: 528-531, 2008.

22. Bongrazio M, Baumann C, Zakrzewicz A, Pries AR and Gaehtgens P: Evidence for modulation of genes involved in vascular adaptation by prolonged exposure of endothelial cells to shear stress. Cardiovasc Res 47: 384-393, 2000.

23. Dixit M, Bess E, Fisslthaler B, et al: Shear stress-induced activation of the AMP-activated protein kinase regulates FoxO1a and angiopoietin-2 in endothelial cells. Cardiovasc Res 77: 160-168, 2008.

24. Park HY, Kang S, Kim GY, et al: Inhibition of neointimal proliferation of rat carotid artery by sulodexide. J Korean Med Sci 12: 210-214, 1997. 